\title{
Flow tilt angles near forest edges - Part 2: Lidar anemometry
}

\author{
E. Dellwik, J. Mann, and F. Bingöl \\ Wind Energy Division, Ris $\emptyset$ National Laboratory for Sustainable Energy, Technical University of Denmark - DTU, \\ P.O. Box 49, 4000 Roskilde, Denmark
}

Received: 3 July 2009 - Published in Biogeosciences Discuss.: 12 August 2009

Revised: 18 January 2010 - Accepted: 14 April 2010 - Published: 26 May 2010

\begin{abstract}
A novel way of estimating near-surface mean flow tilt angles from ground based Doppler lidar measurements is presented. The results are compared with traditional mast based in-situ sonic anemometry. The tilt angle assessed with the lidar is based on 10 or 30 min mean values of the velocity field from a conically scanning lidar. In this mode of measurement, the lidar beam is rotated in a circle by a prism with a fixed angle to the vertical at varying focus distances. By fitting a trigonometric function to the scans, the mean vertical velocity can be estimated. Lidar measurements from (1) a fetch-limited beech forest site taken at $48-175 \mathrm{~m}$ a.g.l. (above ground level), (2) a reference site in flat agricultural terrain and (3) a second reference site in complex terrain are presented.

The method to derive flow tilt angles and mean vertical velocities from lidar has several advantages compared to sonic anemometry; there is no flow distortion caused by the instrument itself, there are no temperature effects and the instrument misalignment can be corrected for by assuming zero tilt angle at high altitudes. Contrary to mast-based instruments, the lidar measures the wind field with the exact same alignment error at a multitude of heights.

Disadvantages with estimating vertical velocities from a lidar compared to mast-based measurements are potentially slightly increased levels of statistical errors due to limited sampling time, because the sampling is disjunct, and a requirement for homogeneous flow. The estimated mean
\end{abstract}

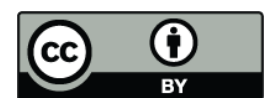

Correspondence to: E. Dellwik (ebde@ risoe.dtu.dk) vertical velocity is biased if the flow over the scanned circle is not homogeneous. It is demonstrated that the error on the mean vertical velocity due to flow inhomogeneity can be approximated by a function of the angle of the lidar beam to the vertical and the vertical gradient of the mean vertical velocity, whereas the error due to flow inhomogeneity on the horizontal mean wind speed is independent of the lidar beam angle. For the presented measurements over forest, it is evaluated that the systematic error due to the inhomogeneity of the flow is less than $0.2^{\circ}$.

The results of the vertical conical scans were promising, and yielded positive flow angles for a sector where the forest is fetch-limited. However, more data and analysis are needed for a complete evaluation of the lidar technique.

\section{Introduction}

Dellwik et al. (2010a) analyzed mean flow tilt angle and vertical velocities from sonic anemometers near a forest edge and found that instrumental precision is severely limited due to flow distortion. Especially at the highly turbulent forested sites, eddies hit the anemometer at steep angles of attack for which it is usually not calibrated. Since the attack angle distribution on the sonic anemometer is non-symmetric with steeper attack angles from below than from above (Raupach et al., 1996), flow distortion errors from updrafts and downdrafts do not even out. If flow tilt angles should be related to features in the terrain such as a forest edge, another issue affecting the measurement precision concerns the vertical alignment of the anemometer. 
In this study, we explore the possibility of using ground based remote sensing wind lidars (LIght Detection And Ranging) for mean flow tilt angle estimation. The lidar technique has the advantage that it is flow-distortion free and builds on simple and fundamental physical principles. It also enables measurements at several distances from the ground in the exact same coordinate system. This last feature could be used to correct for instrument misalignment, by applying an assumption that far above the surface, mean flow tilt angles are negligible.

Whereas sonic anemometry has been extensively used for determining mean vertical velocities, the lidar anemometry is a novel technique. Lasers have been used extensively to measure fluctuations of the vertical velocity (Lothon et al., 2006; Davis et al., 2009; Mann et al., 2009), but the mean vertical wind has never been reported. For the type of lidar used in this study, the mean vertical velocity is calculated from conical scans of velocities along the beam direction, and the beam is focused at predefined heights. The mean wind speed field can then be estimated from the scans. However, if the terrain is not homogeneous and the flow field varies over the cone, the derived velocities will be biased.

Ground-based remote sensing wind lidars are in rapid development, due to technology improvements of lasers and other optical components used in the telecommunications industry. The development is driven by the needs of the wind energy industry to precisely estimate wind resources typically in the range of $80-150 \mathrm{~m}$ above the surface. The lidar in focus for this study has been extensively tested since high precision is needed for wind energy applications. For example, Smith et al. (2006) reported that the instrument reproduces the horizontal mean wind speed at all the investigated heights within a few percents, but can experience problems when the distribution of backscatter is very inhomogeneous, as in the case of low clouds or fog (Courtney et al., 2008). Over the ocean the instrument has been used for wind resource estimation (Kindler et al., 2007; Pena et al., 2008a) and wind profile measurements (Pena et al., 2008b) showing very good agreement with cup anemometer up to $160 \mathrm{~m}$. For the conically scanning mode, which is used to derive the horizontal wind speed, horizontal homogeneity is assumed. However, as we shall see later, for measurements over ordinary terrain this may not be a valid assumption, as pointed out by Bingöl et al. (2009), and the error committed on the horizontal mean wind speed may be up to $10 \%$ depending on the steepness and complexity of the terrain. A second application of the instrument in wind energy has been the investigation of flow around a wind turbine (Harris et al., 2007; Bingöl et al., 2010).

Precise measurements of the flow tilt angle and the mean vertical velocity $W$ in the lower as well as upper part of the atmospheric boundary layer are important for several reasons. In the lower part, i.e. in the surface layer, correct estimation of mean tilt angle is crucial for understanding the wind flow at obstacles, forests edges and in complex terrain.
The mean flow angle is of practical importance for operating wind turbines, where angles different from zero may lead to suboptimal performance. Another application concerns the estimation of the vertical advection term for the surface carbon balance (Lee, 1998). In the upper part of the atmospheric boundary layer, $W$ is a modulator of convection, since areas of rising air increase convection and areas of sinking air suppress convection (Lenschow et al., 2007). Also, for the understanding and correct modeling of the height of the atmospheric boundary layer, which in turn is an important parameter for many surface layer processes, correct quantification of entrainment processes are important. However, both in the upper and lower part of the atmospheric boundary layer, the measurement of $W$ and mean tilt angle poses an extreme challenge for experimentalists due to its generally small magnitude.

\section{Theory}

\subsection{Vertical velocity measured by a homodyne lidar in the conically scanning mode}

Wind lidars utilize the Doppler shift of light emitted by a laser and scattered back by aerosols to calculate the wind speed in the direction of the beam. The technique is calibration-free and relies only on the detection of the laser light frequency shift. Currently, there are several wind lidars available commercially, but in this study the scope was limited to the ZephIR lidar (Natural Power, UK), which was the first to address the wind energy market. The ZephIR lidar calculates the mean wind field by rotating the laser beam at a fixed half-opening angle $\varphi=30.4^{\circ}$, such that the surface scanned by the lidar beam has the shape of a cone. This mode of measurement is called conical scanning mode and is also common for radar measurements (Frisch et al., 1989). The ZephIR lidar is a continuous wave wind lidar, which determines the measurement range by focusing of the laser beam. As a result, the volume over which the wind speed is measured is increasing quadratically with height (Sonnenschein and Horrigan, 1971; Smith et al., 2006). Mathematically, the unit vector in the direction of the laser beam is given by

$\boldsymbol{n}=(\cos \theta \sin \varphi, \sin \theta \sin \varphi, \cos \varphi)$,

where $\theta$ is the azimuthal direction of the beam. The projection of a homogeneous mean wind field $\boldsymbol{U}=(U, V, W)=$ $\left(U_{h} \cos \theta_{0}, U_{h} \sin \theta_{0}, W\right)$, where $U_{h}=\sqrt{U^{2}+V^{2}}$ and $\theta_{0}$ is the mean wind direction, onto $\boldsymbol{n}$ yields an expression for the lineof-sight velocity $v_{\text {los }}$ :

$v_{\text {los }}(\theta)=U_{h} \cos \left(\theta-\theta_{0}\right) \sin \varphi+W \cos \varphi$.

With the ZephIR lidar, the Doppler shift of the light is measured using homodyne detection, which only enables the determination of the absolute value of the Doppler shift and not the sign. Hence only absolute values of the velocity can 

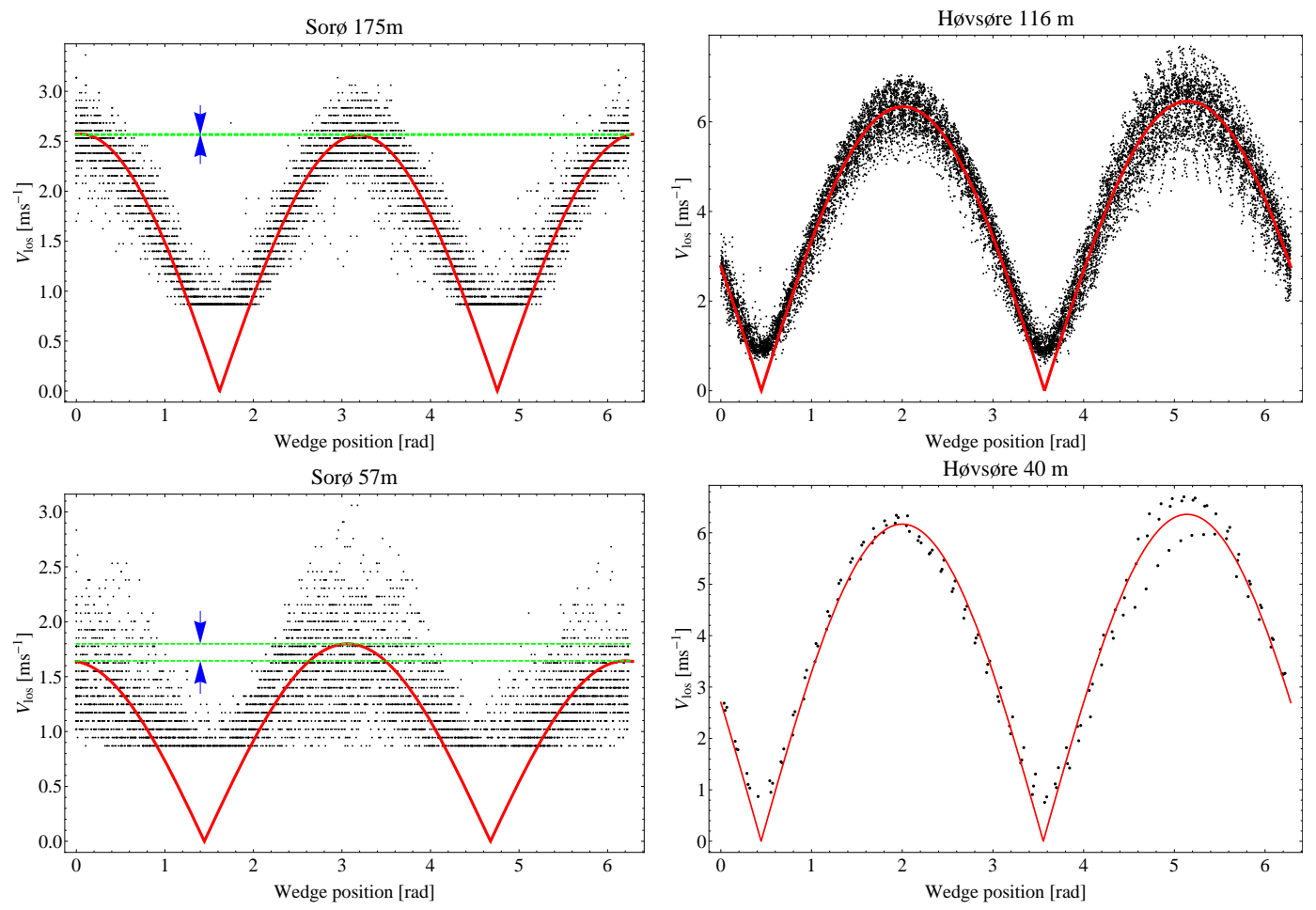

Fig. 1. Examples of lidar line-of-sight velocities from conical scanning mode. The two plots from Sorø and the $116 \mathrm{~m}$ plot from Høvsøre show the line-of-sight velocities for a $30 \mathrm{~min}$ period and the $40 \mathrm{~m}$ Høvsøre plot illustrate data from a $3 \mathrm{~s} \mathrm{scan}$. The peaks of the fit (Eq. 3 , thick red line) define the wind speed in the mean wind direction, and the difference in the peak heights show the mean vertical velocity for homogeneous flow.

be measured. Examples of velocities measured in the conical scanning mode are given in Fig. 1. In the figure, each point represents a mean value of 4000 Doppler spectra, from which $v_{\text {los }}$ is determined. The wind field is determined from fitting a trigonometric function (full line) to the measured $v_{\text {los }}(\theta)$;

$y=\left|A \cos \left(\theta-\theta_{0}\right)+B\right|$.

Because of the homodyne detection of the Doppler shift, there is a sign ambiguity in the wind vector in Eq. (3), which affects both the mean wind direction $\left( \pm 180^{\circ}\right)$ and the sign of $W$. The ambiguity is removed in the data processing by an external determination of the wind direction from a wind vane as described in Smith et al. (2006). Once the wind direction is determined, the sign of $W$ is also determined. When the ambiguity is removed, $U_{h}$ and $W$ can easily be determined by equating Eqs. 2 and 3. In Fig. 1 (bottom left), the arrows indicate the offset $B=W / \cos \varphi$. The mean flow tilt angle is subsequently calculated as

$\tan \alpha=\frac{W}{U_{h}}$.

A conspicuous feature in Fig. 1 is that there is a threshold around $1 \mathrm{~ms}^{-1}$ below which no measurements are recorded. This threshold is introduced to avoid excessive scatter at low wind speeds since the background noise of the laser obscures the signal. This noise is called RIN, which is an abbreviation of Relative Intensity Noise (Siegman, 1986, Sect. 25.1), and is an unfavorable property of the laser.

In Sect. 5, the possibility to determine mean flow tilt angles using another type of lidar is discussed.

\subsection{Lidar error caused by inhomogeneous mean flow}

Equation (4) is only valid if the mean wind flow is horizontally homogeneous, but here we allow for a linear variation in space. This approach was also used in Browning and Wexler (1968). Without loss of validity, the following analysis is ignoring the fact that the lidar only measures the magnitude of the wind speed in the direction of the beam, not the sign.

Assume the mean wind field $\boldsymbol{U}=(U, V, W)=\left(U_{1}, U_{2}, U_{3}\right)$ over a $10-30 \mathrm{~min}$ averaging period to vary linearly

$U_{i}(\boldsymbol{x})=U_{i}(\mathbf{0})+x_{j} \frac{\partial U_{i}}{\partial x_{j}}$

over a volume enclosing the lidar scanning circle. The origo of the coordinate system $\boldsymbol{x}=\mathbf{0}$ is the center of the scanning circle elevated by $h$ over the instrument. In a homogeneous flow field, the along beam wind speed component, $v_{\text {los }}$, 
measured at an azimuthal angle $\theta$ is the projection of $\boldsymbol{U}$ onto $n$ (Eq. 1):

$v_{\operatorname{los}}(\theta)=\boldsymbol{n}(\theta) \cdot \boldsymbol{U}(\boldsymbol{n}(\theta) l-(0,0, h))$,

where the velocity field is evaluated in the position of the focus of the laser beam. Here $l=h / \cos \varphi$ is the focus distance. The additional variations of $v_{\text {los }}$ due to lack of homogeneity may be expressed as $v^{\prime}$ los $(\theta)=v_{\text {los }}(\theta)-\boldsymbol{n}(\theta) \cdot \boldsymbol{U}(\mathbf{0})$, and it can be written in terms of the velocity gradient:

$v_{\text {los }}^{\prime}(\theta)=n_{i}(\theta)\left(n_{j}(\theta) l-\delta_{j 3} h\right) \frac{\partial U_{i}}{\partial x_{j}}$.

Substituting Eq. (1) into this equation and ordering the terms as a Fourier series in $\theta$ we finally get

$$
\begin{aligned}
& v_{\text {los }}(\theta)=W \cos \varphi+\frac{l}{2} \sin ^{2} \varphi \overbrace{\left(\frac{\partial U}{\partial x}+\frac{\partial V}{\partial y}\right)}^{=-\partial W / \partial z} \\
& +\sin \varphi\left(U+l \cos \varphi \frac{\partial W}{\partial x}\right) \cos \theta \\
& +\sin \varphi\left(V+l \cos \varphi \frac{\partial W}{\partial y}\right) \sin \theta \\
& +\frac{l}{2} \sin ^{2} \varphi\left(\frac{\partial U}{\partial x}-\frac{\partial V}{\partial y}\right) \cos 2 \theta \\
& +\frac{l}{2} \sin ^{2} \varphi\left(\frac{\partial U}{\partial y}+\frac{\partial V}{\partial x}\right) \sin 2 \theta .
\end{aligned}
$$

The horizontal and vertical wind speeds are derived from the lidar measurements by fitting a trigonometric series $a+b \cos \theta+c \sin \theta$, which is equivalent to Eq. (3), to the data. The vertical wind speed is then estimated as $a / \cos \varphi$ while the horizontal components are $b / \sin \varphi$ and $c / \sin \varphi$, respectively. In the presence of a linear deviation from homogeneity we thus get for the wind vector estimated from the lidar:

$$
\begin{aligned}
U_{\text {lidar }} & =U+h \frac{\partial W}{\partial x} \\
V_{\text {lidar }} & =V+h \frac{\partial W}{\partial y} \\
W_{\text {lidar }} & =W-\frac{h}{2} \tan ^{2} \varphi \frac{\partial W}{\partial z} .
\end{aligned}
$$

Equation (11) was obtained by isolating all terms in Eq. (8) that were independent of $\theta$ and then dividing the result with $\cos \varphi$. Since the constant terms, the $\cos n \theta(n=1,2)$ and $\sin n \theta$ terms are orthogonal, the fact that the $\cos 2 \theta$ and $\sin 2 \theta$ term in the fitting procedure (Eq. 11), will not add to the bias of $W$ caused by the linearly varying flow field. The omittance of these terms may however result in a worse fit by Eq. (3) and contribute to a random error on $W$.

The error in $W$ caused by the inhomogeneity will vanish for high altitudes, since $0.5 h \partial W / \partial z$ approaches zero monotonously with height.
As a result of these equations, the error due to inhomogeneity of the mean flow will disappear for the vertical component as the half opening angle $\varphi$ goes to zero, while the errors on the horizontal components are independent of $\varphi$.

\section{Materials and methods}

The goal of this study is to estimate the flow tilt angles at a flat forested site described below. However, since the method of determining $W$ from a lidar has not been tested before and the mean flow tilt angles at the forest site are expected to be small (Dellwik et al., 2010a), two reference sites were included in the analysis.

\subsection{Sites}

Three Danish sites were investigated; the forest site Sorø $\left(55^{\circ} 29^{\prime} 9.5^{\prime \prime} \mathrm{N} 11^{\circ} 38^{\prime} 40.5^{\prime \prime} \mathrm{E}\right)$, the steep terrain site Bolund $\left(55^{\circ} 42^{\prime} 13.0^{\prime \prime} \mathrm{N} 12^{\circ} 05^{\prime} 51.9^{\prime \prime} \mathrm{E}\right)$ and the coastal flat site at Høvsøre $\left(56^{\circ} 26^{\prime} 25.80^{\prime \prime} \mathrm{N} 8^{\circ} 9^{\prime} 2.9^{\prime \prime} \mathrm{E}\right)$. The Sorø site is described in detail in Dellwik et al. (2010a). It is a typical Danish beech forest of limited extent $(\approx 2 \times 1 \mathrm{~km})$ containing clearings. At the time of the experiment, the trees were about $26-27 \mathrm{~m}$ tall. The mast at the site is $57 \mathrm{~m}$ and $8 \mathrm{~m}$ south of the mast, a scaffolding tower of $24 \mathrm{~m}$ height is located. The lidar was placed on the scaffolding tower, such that no trees shadowed the lidar beam. A Solent R2 (Gill Instruments Ltd.) was mounted at $48 \mathrm{~m}$ height in the mast, thereby matching the lowest focus distance of the lidar.

The Høvsøre site represents a very flat site with low vegetation (Fig. 2), where zero mean flow tilt angles are expected. Høvsøre is situated $1.5 \mathrm{~km}$ from the North Sea on the west coast of Denmark in a rural area. The site has seven masts ranging from around $70 \mathrm{~m}$ to $160 \mathrm{~m}$ equipped with anemometers and other meteorological sensors measuring temperature, pressure, precipitation etc. The southernmost mast is the most well equipped with cup anemometers at 10, 40, 60, 80,100 , and $116.5 \mathrm{~m}$ and USA- 1 scientific sonic anemometers (Metek $\mathrm{GmbH})$ at 10, 20, 40, 60, 80, and $100 \mathrm{~m}$. The cup and sonic anemometer data were stored almost continuously at $10 \mathrm{~Hz}$ and $20 \mathrm{~Hz}$, respectively, since 2004 . The lidars investigated here were located close to the mast. More information about the site may be found in Smith et al. (2006); Sjöholm et al. (2009).

The Bolund site is located on a peninsula, where flow angles should vary due to the steep terrain slopes. The hill is covered with low grass. It is $12 \mathrm{~m}$ high and extends $130 \mathrm{~m}$ in the W-E direction and $75 \mathrm{~m}$ in the N-S direction (Fig. 3). During the Bolund experiment, ten masts were operated. The lidar was located $8.5 \mathrm{~m}$ to the south of mast 2 near the edge of the escarpment. Mast 2 was instrumented with five USA-1 basic anemometers (Metek GmbH.) at 1.1, 2.1, 3.6, 5.1 and $9.1 \mathrm{~m}$ height as well as two cup anemometers at 9.1 and $11.1 \mathrm{~m}$ height. The lidar position and measurement heights 

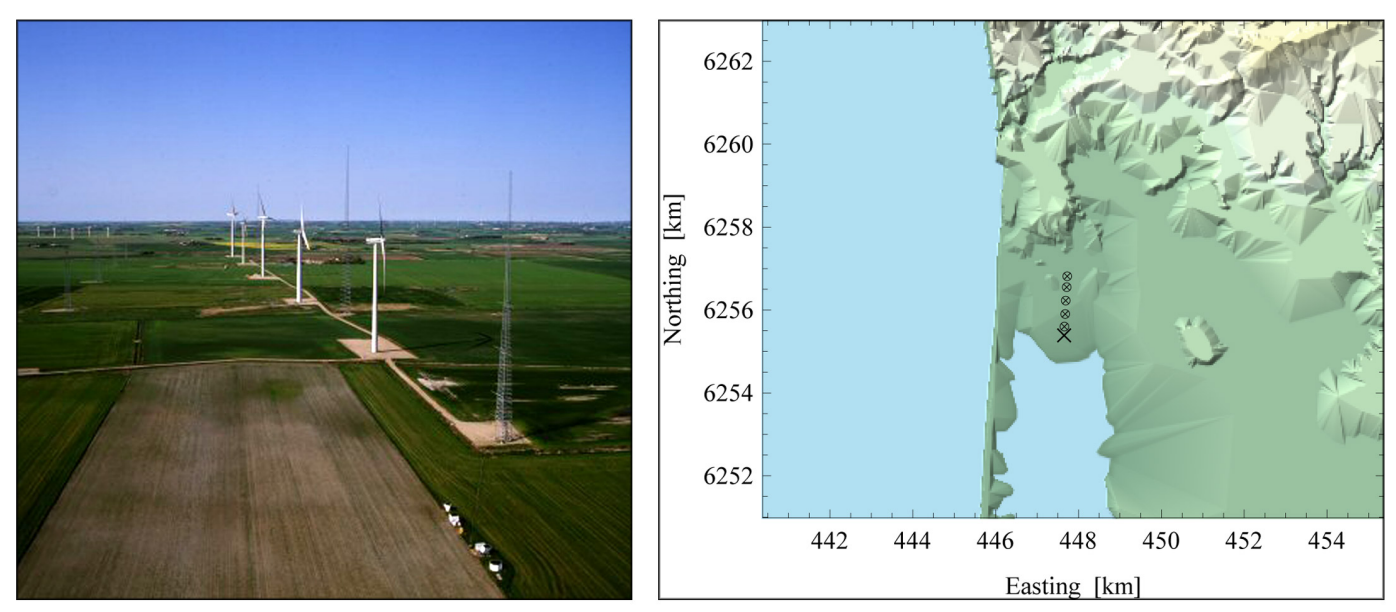

Fig. 2. Photo and map of the Høvsøre site. In the foreground of the photo, a row of lidars and sodars can be seen. However, the lidar utilized in this study was located approximately $20 \mathrm{~m}$ to the north of the mast. The highest point of the map represents a height of $50 \mathrm{~m}$, whereas the mast (cross) and turbines (circles) are located only a few meters above sea level.
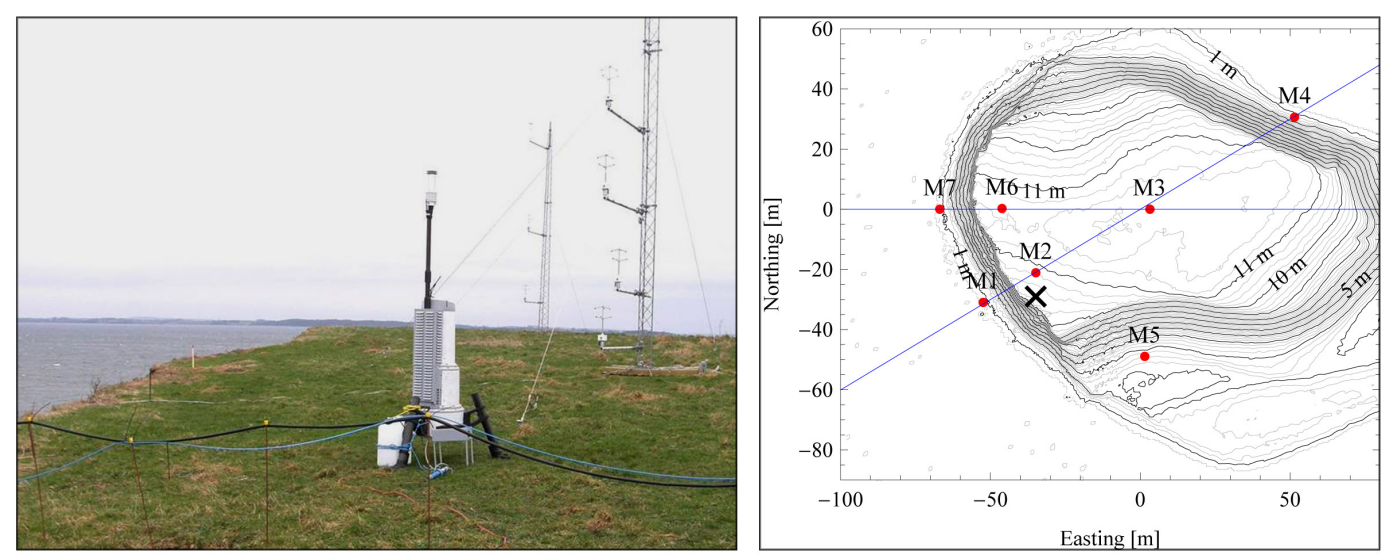

Fig. 3. Photo of the ZephIR lidar (left) and map with masts positions at the Bolund experiment (right). In the background of the photo, masts 2 and 6 can be seen. Mast 2 was located $9 \mathrm{~m}$ from the western escarpment. The ZephIR lidar was located at the cross in the map, $8.5 \mathrm{~m}$ to the south of mast 2 and only $3 \mathrm{~m}$ from the escarpment.

changed during the approximately two-month long campaign. Also, instrumental errors occurred, and the longest continuous measurement series with a very high measurement height $(300 \mathrm{~m})$ only covered five days. More information on the Bolund experiment can be found in Bechmann et al. (2009).

\subsection{Lidar instrumentation}

For this study, field campaign lidar data from the three sites were analyzed. The first experiment took place at the Sor $\varnothing$ site, where the prototype of the ZephIR was used, whereas at the two experiments at Bolund and Høvsøre, the commercial version of the instrument was used. The prototype differs from the commercial version in that the optical head containing the focusing system and the rotating wedge is separated from the processing unit by an optical cable, thereby allowing for more flexible use since the less bulky and lighter optical head can be mounted in a variety of positions. The noise level of the prototype is slightly higher than in the commercial version.

The durations of the experiments, measurement heights and lidar types are listed in Table 1. Both versions of the ZephIR measure three revolutions at each measurement height, where each revolution takes about one second. The shift between heights also takes about $1 \mathrm{~s}$. A whole measurement cycle therefore takes about $n \times 4 \mathrm{~s}$ where $n$ is the number of measurement heights.

After completing the experiment listed in Table 1, the optical head was for a shorter period mounted on the mast measuring horizontally. The prototype was focus calibrated at Risø-DTU. 
Table 1. Overview of lidar experiments.

\begin{tabular}{llll}
\hline Site & Lidar & Duration of experiment & Measurement height (m) \\
\hline Sorø & ZephIR prototype & 15 Nov 2006-6 Jan 2007 & 48, 57, 65, 76, 92, 113, 175 \\
Høvsøre & ZephIR Commercial & 8 Jan 2009-2 Nov 2009 & 40, 60, 80, 100, 116 \\
Bolund & ZephIR Commercial & 1 Feb 2008-5 Feb 2008 & 11, 20, 50, 100, 300 \\
\hline
\end{tabular}

\subsection{Data processing}

The Solent R2 and USA-1 basic sonic anemometers were corrected for flow distortion in the same way as was described in Dellwik et al. (2010a). The USA-1 Scientific anemometers at Høvsøre have a built-in flow distortion correction identical to the one that was applied for the USA1 Basic in the post-processing. These corrections and their limitation were described in Dellwik et al. (2010a).

For all sites a minimum wind speed of $3 \mathrm{~ms}^{-1}$ was used, to ensure (a) that the signal is above the RIN level and (b) that the mean wind direction was well-defined. Initially, the minimum wind speed requirement was investigated, where it was found that the $3 \mathrm{~ms}^{-1}$ limit was satisfactorily as long as the initial guesses for the constants in the non-linear fits of lineof-sight velocities (Eq. 3 and Fig. 1) were sufficiently accurate. The presented results did not depend strongly on the value of this minimum velocity.

For the Høvsøre lidar data, it was also required that at every three second measurement cycle, the number of valid measurements of the line-of-sight velocities should be larger than 100 , and that the fit of the rectified cosine function to the data should have some maximum averaged deviation. The validity of a measurement is determined using an algorithm implemented by the manufacturer. The reason for the requirement of more than 100 valid measurements was to avoid possibly erroneous samples due to, for example, lack of aerosols in the air. The Høvsøre analysis was also done without this constraint, which resulted in only a few extra outliers and almost the same median flow tilt angle. At Bolund, all lidar data were used, since the mean wind speed was always larger than $3 \mathrm{~ms}^{-1}$ during the period, and the instrument worked well. Both at Bolund and Høvsøre, the $30 \mathrm{~min}$ vertical velocity was obtained by averaging all three second vertical velocities in that period. This corresponds to using the fits in Fig. 1 (bottom right), which is the standard output from the commercial lidar.

At Sorø, the prototype was working less reliably and we chose, for each half hour period, to collect all line-of-sight velocities for a given height and do the fit to the rectified cosine function with all these data. Examples of fits at a low wind speed $\left(U=3.4 \mathrm{~ms}^{-1}\right.$ at $\left.57 \mathrm{~m}\right)$ are given in Fig. 1 (left), where the horizontal stripes are caused by discrete values of the line-of-sight velocity.

\section{Results}

\subsection{The flat terrain reference site}

The data shown in Fig. 4 (top) are 30 min averages of the vertical velocity measured by the ZephIR close to the meteorological mast at Høvsøre as a function of wind direction. Wind coming from the north may be disturbed by the five large turbines on the test stand, but between the gray vertical lines the influence from the turbines should be negligible. A misalignment of the instrument with respect to the vertical direction gave a spurious sinusoidal variation of the mean flow tilt angle with direction. Such a variation with an amplitude of $0.5^{\circ}$ was observed for the highest focus distance and was subtracted from all focus distances. Subsequently, the mean vertical wind speed was close to zero for all directions and heights, and the scatter around zero on the half hour values is between $0.25^{\circ}$ and $0.5^{\circ}$.

In Fig. 4 (bottom), the corresponding mean flow tilt angles at 40 and $100 \mathrm{~m}$ from the sonic anemometers are shown. They have an off-set of $-1.5^{\circ}(40 \mathrm{~m})$ and $-0.5^{\circ}(100 \mathrm{~m})$, and the $40 \mathrm{~m}$ anemometer seems to show a misalignment of about $1.5^{\circ}$.

The scatter, which is an estimate of the statistical error, from the sonic anemometers and the lidar is of similar size, despite the fact that the sampling of the lidar is disjunct and in absolute time only covers $30 / n$ minutes at each sampling height.

\subsection{The complex terrain reference site: Bolund}

A comparison of mean flow tilt angles from the lidar and the $9.1 \mathrm{~m}$ sonic anemometer as a function of wind direction is shown in Fig. 5. During the five-day lidar experiment, wind directions were predominantly from South-West. Both the $300 \mathrm{~m}$ and $100 \mathrm{~m}$ focus distances show flow angles very close to zero, and the influence from the Bolund escarpment (western edge) is clearly visible for the lower focus distances. The shape of the wind direction response is similar for the lidar and the $9.1 \mathrm{~m}$ sonic anemometer, but the tilt angles measured by the sonic anemometer are considerably smaller in the interval $\left[160^{\circ}, 290^{\circ}\right]$ with a maximum around $4.5^{\circ}$ compared to around $9^{\circ}$ for the lidar at $11 \mathrm{~m}$ focus height.

Over the hill, the tilt angles decrease with height and using Eq. (11) with $\partial W / \partial z<0$, the lidar would overestimate 

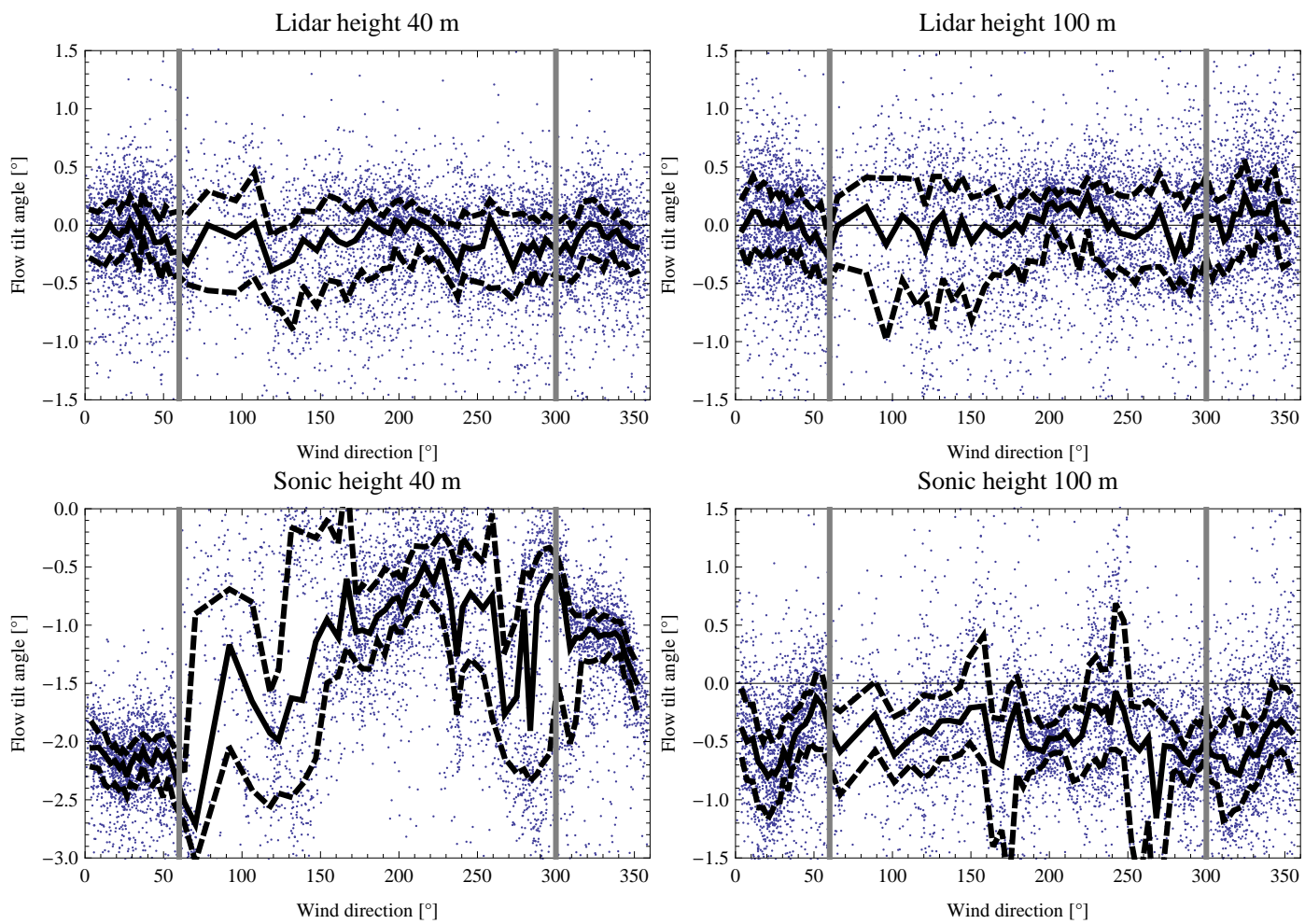

Fig. 4. Comparison of lidar (top) and sonic (bottom) mean flow tilt angles $(\alpha)$ at Høvsøre using all data. Each point in the graphs signifies a 30 min mean value, the full line is the median and the dashed lines represent the $25 \%$ and $75 \%$ quartiles.
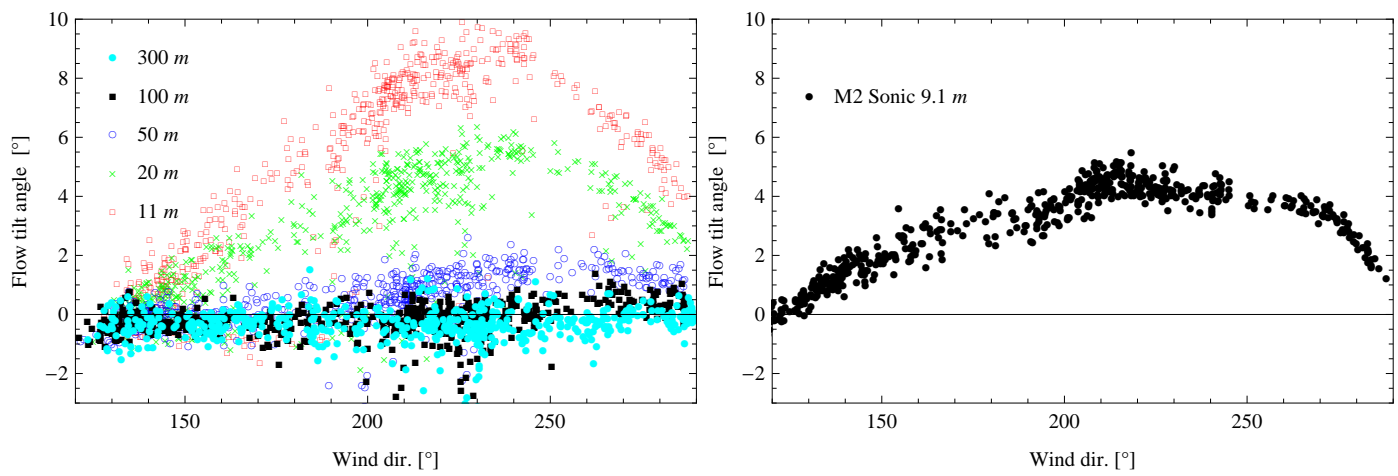

Fig. 5. Comparison of lidar (left) and sonic (right) 30 min mean flow tilt angles at Bolund using all data.

the flow angle as a result of the inhomogeneity of the flow. However, the mismatch can also be explained by the proximity to the edge; since the sonic anemometer was located about three times further from the edge, the lidar flow angles should be considerably larger. In Sect. 5, the error due to the inhomogeneity of the flow is estimated.

\subsection{The forest site: Sorø}

Mean flow tilt angles as a function of wind direction during the several months long campaign at the Sorø site are shown in Fig. 6. Because of a limitation of the variation in wind directions during the experiment, it was hard to deduce whether the lidar was slightly tilted with respect to vertical. As for the Bolund results, the flow tilt angles decrease monotonously with height. In Fig. 7, 30 min mean values both from the lidar (top) and the two sonic anemometers (bottom) are shown. At the $48 \mathrm{~m}$ level, the coincidence between the median curves of the lidar and the sonic anemometer is rather good. However, the $43 \mathrm{~m}$ anemometer shows negative tilt angles for the same direction interval. The scatter around the median from the lidar was higher than for the sonic anemometer, probably 


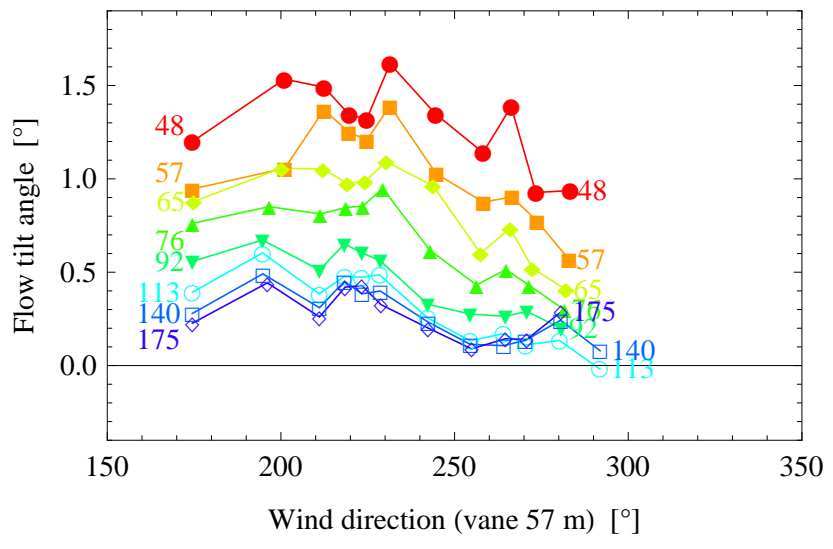

Fig. 6. Mean flow tilt angles measured by lidar at Sorø, all measurement heights.

reflecting the effect of the disjunct sampling in the very turbulent forest flow.

The positive flow angles can be explained by a decelerating flow and a growing internal boundary layer (Dellwik et al., 2010a).

\section{Discussion}

\subsection{Assessment of lidar accuracy for measuring flow tilt angles and mean vertical velocities}

None of the lidar experiments presented here was designed to measure mean flow tilt angles, but rather to assess mean wind speeds and directions as well as momentum fluxes. Since the lidar and the mast was not co-located, the comparison between sonic anemometer and lidar is not straight-forward at the Bolund site. Both terrain effects and fundamental measurement issues caused by flow inhomogeneity could explain the mis-match in the mean directional response. To estimate the error; the flow tilt angle error due to inhomogeneity of the mean flow field was estimated as

$\alpha_{\mathrm{err}}=-\frac{h}{2} \tan ^{2} \varphi \frac{\partial \alpha}{\partial z}$.

where Eq. (11) together with the small angle approximation $\tan \alpha \approx \alpha$ was used. Equation (12) was evaluated to approximately $+1^{\circ}$ at the $11 \mathrm{~m}$ focus distance at the Bolund site, using the difference between the flow angles at $11 \mathrm{~m}$ and $20 \mathrm{~m}$. This is a conservative estimate since we evaluate the difference on the measured data (which include the error) and the measured gradient is slightly higher than the true gradient. Hence, it can be concluded for the Bolund results, that the mismatch of the sonic anemometer at $9.1 \mathrm{~m}$ and the lidar in Fig. 5 was mainly caused by the different positions and measurement height of the ZephIR lidar and the met mast 2.

Since the tilt angles are small at the Sor $\varnothing$ site, it was assumed that $\alpha / z \approx \partial \alpha / \partial z$, and using Eq. (12), $\alpha_{\text {err }}$ was estimated to $-0.13^{\circ}$ from the real $\alpha$ for the $48 \mathrm{~m}$ measurement. The error could also be estimated from the tilt angle difference between 57 and $48 \mathrm{~m}$ to $+0.18^{\circ}$.

From the Høvsøre experiment, the ZephIR lidar systematic error, defined as the difference between zero and the measured flow angle at $100 \mathrm{~m}$, is for any wind direction sector less than $0.3^{\circ}$.

The statistical error due to limited sampling time, was ranging from $0.5^{\circ}$ for the $\mathrm{H} \varnothing \mathrm{v} \varnothing$ re experiment up to about $1^{\circ}$ for the lowest measurement height at the Sorø forest. These estimates could be expected to be lower for sonic anemometers due to the effect of disjunct sampling of the lidar data. The good agreement between the $48 \mathrm{~m}$ sonic anemometer and lidar data in Fig. 7 is regarded as coincidental, because of the great uncertainties associated with sonic anemometer flow distortion and alignment (Dellwik et al., 2010a) and the discrepancy with the measurements at $43 \mathrm{~m}$.

For the presented data, the vertical alignment was determined from the assumption that at the highest measurement level, the flow tilt angle is zero. Another possibility would be to improve the alignment of the instrument itself by using high-accuracy leveling devices.

\subsection{Other possibilities for utilizing lidars for surface layer flow tilt angles}

The presented study is devoted to the ZephIR lidar. Since this lidar is affected by RIN, it is difficult to measure at very low mean wind speeds. If the lidar is pointed directly vertically, RIN would drown the signal and besides, the sign ambiguity would not be resolved, which would render the signal useless for mean vertical velocity estimation. However, the development of laser technology is rapid and already as of today, RIN can essentially be eliminated.

Another possibility to measure $W$ with ground-based lidar would be to use the WindCube, which is a heterodyne pulsed lidar produced by Leosphere, France (Courtney et al., 2008). This lidar does not suffer from RIN, and because of heterodyning, the sign ambiguity can be resolved without using a wind vane. The fact that it is pulsed and measure at all heights simultaneously implies that there should be no effect of increased scatter due to disjunct sampling. Yet another possibility is the Galion lidar (Sgurr Energy, UK). This lidar is also heterodyne and pulsed, but has in addition flexible mirror-based scanning head that can do conical scanning as well as staring directly upwards. These possibilities should be investigated further in order to assess more completely the potential of the lidar for mean vertical velocity and flow tilt angles measurements.

A disadvantage of the pulsed lidar systems, is that the lowest possible measurement height is in the order of 40 to $60 \mathrm{~m}$, whereas the continuous wave ZephIR lidar can measure down to $10 \mathrm{~m}$ and even further down with simple modifications. 

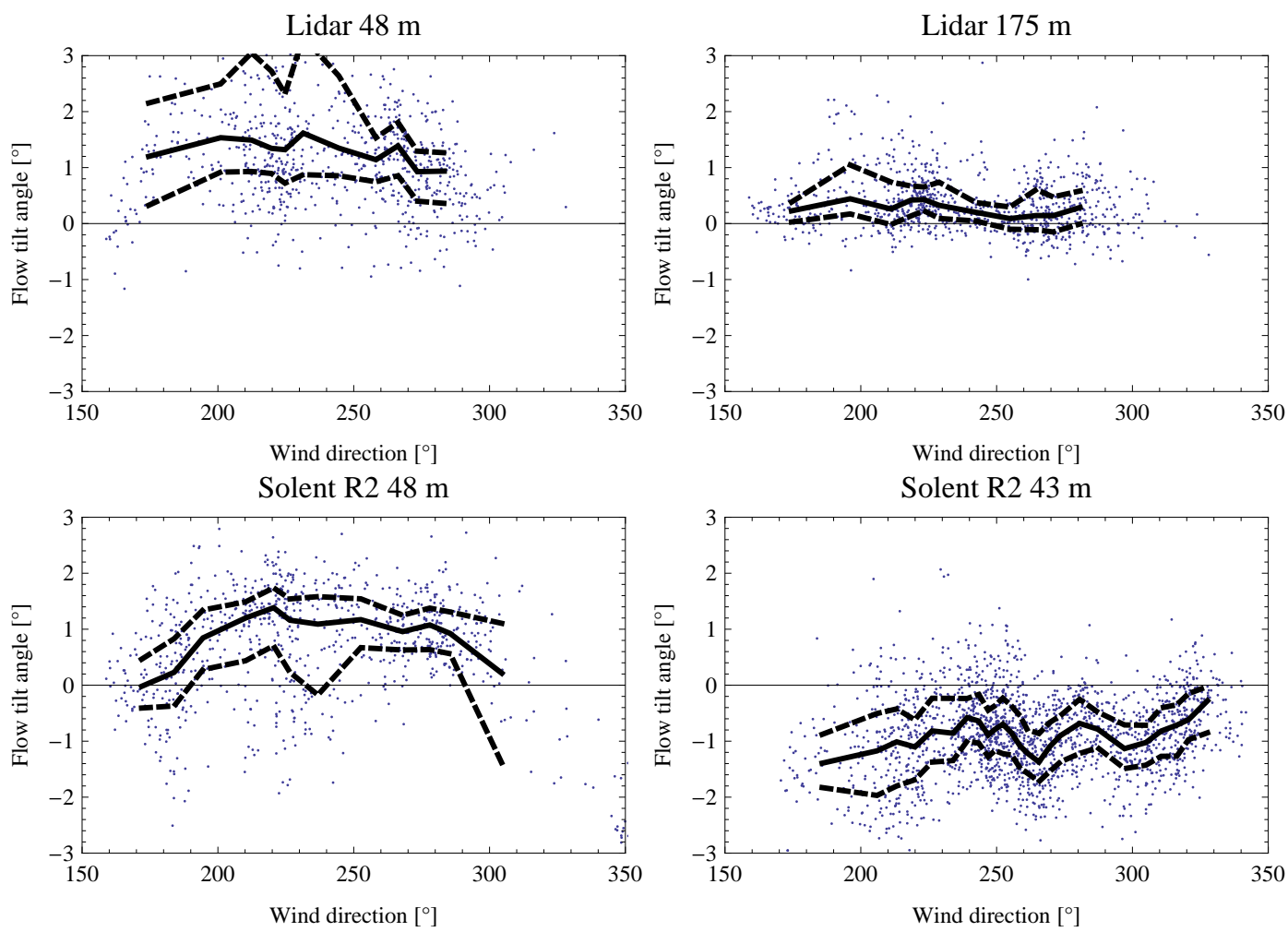

Fig. 7. Lidar and sonic comparison from Sorø. There is only one overlapping measurement height $(48 \mathrm{~m})$.

\section{Conclusions}

The ZephIR Doppler wind lidar was tested for its ability to measure mean flow tilt angles. The motivation for this study was to evaluate flow tilt angles in a very turbulent forest flow, where flow distortion constitute at severe limitation on sonic anemometer accuracy (Dellwik et al., 2010a). Since the mean flow tilt angles over the forest are expected to be small, the method was evaluated at two reference sites: the very flat site Høvsøre and the steep hill site Bolund. At the Høvsøre site, the lidar measured tilt angles very near zero, whereas the two sonic anemometers used for comparison showed a negative off-set and - one of them - an effect of misalignment. The systematic error of the Høvsøre lidar was evaluated to much less than $0.5^{\circ}$. The scatter around the median, which reflects the statistical error due to limited sampling time, was similar for sonic anemometers and the lidar. For the Bolund site, flow angles up $10^{\circ}$ was measured with the lidar. The high flow angles were measured in the wind direction where the flow is the most inhomogeneous. By using the measured lidar gradient, the systematic error due to the flow inhomogeneity was estimated to $+1^{\circ}$. The Bolund sonic anemometer at the most similar height as the lowest lidar focus distance measured flow angles almost half the magnitude as the lidar. This discrepancy could mainly be explained by the lidar being located closer to the Bolund escarpment. Finally at the Sorø forest site, where the ZephIR prototype was employed, the lidar measured positive flow angles of around $1.5^{\circ}$ at the lowest measurement height $(48 \mathrm{~m})$ and between $0.1^{\circ}$ and $0.4^{\circ}$ at the highest measurement level $(175 \mathrm{~m})$. The systematic error due to the flow inhomogeneity was estimated to less than $0.2^{\circ}$, where the sign of the error could not be determined. The two sonic anemometers at the Sorø site measured positive and negative flow angles, respectively. The high level of turbulence at the forest site was reflected in higher statistical uncertainty. We conclude that lidar anemometry can provide consistent estimates of mean flow tilt angles also for the very turbulent forest flow. In general, the results from all sites pointed to the high accuracy of the lidar. Since this is the first study concerning the use of the vertical mean wind speed, more evaluation of the lidar technique for measuring the mean vertical component of the wind is needed. 
Acknowledgements. This work is financed through the UPWIND project (WP6) funded by the European Commission, the NECC project funded by the Nordic Natural Science Research Councils (NOS-N) and the Vindkraft i skog project financed by Elforsk, Sweden. We are also grateful for the support provided from the projects windscanner.dk, Center for computational wind turbine aerodynamics and atmospheric turbulence and the Forest and Wind Profile funded by the Danish Strategic Research Council. Use of lidar data from the NorseWind project taken at the Høvsøre site was kindly granted by Andy Oldroyd. The Bolund experiment was funded by the Danish Energy Agency through contract ENS-33033-0062.

Edited by: A. Lindroth

\section{References}

Bechmann, A., Berg, J., Courtney, M., Jørgensen, H., Mann, J., and Sørensensen, N.: The Bolund Experiment: Overview and Background, Tech. Rep. Ris $\varnothing-\mathrm{R}-1658(\mathrm{EN}), \mathrm{Ris} \emptyset \mathrm{DTU}-\mathrm{Na}-$ tional Laboratory for sustainable energy, 2009.

Bingöl, F., Mann, J., and Foussekis, D.: Modeling conically scanning lidar error in complex terrain with WAsP Engineering, Meteorol. Z., 18, 189-195, 2009.

Bingöl, F., Mann, J., and Larsen, G.: Lidar Measurements of Wake Dynamics Part I: One Dimensional Scanning, Wind Energy, 13, 51-61, 2010.

Browning, K. A. and Wexler, R.: The Determination of Kinematic Properties of a Wind Field Using Doppler Radar, J. Appl. Meteorol., 7, 105-113, 1968.

Courtney, M., Wagner, R., and Lindelöw, P.: Testing and comparison of lidars for profile and turbulence measurements in wind energy, http://stacks.iop.org/1755-1315/1/012021, last access: 20 May 2010, IOP Conference Series: Earth and Environmental Science, 1, 012021, 14 pp., 2008.

Davis, J. C., Collier, C. G., Davies, F., Pearson, G. N., Burton, R., and Russell, A.: Doppler lidar observations of sensible heat flux and intercomparisons with a ground-based energy balance station and WRF model output, Meteorol. Z., 18, 155-162, 2009.

Dellwik, E., Mann, J., and Larsen, K.: Flow tilt angles near forest edges - Part 1: Sonic anemometry, Biogeosciences, 7, 17451757, doi:10.5194/bg-7-1745-2010, 2010.
Frisch, A. S., Martner, B. E., and Gibson, J. S.: Measurement of the vertical flux of turbulent kinetic energy with a single Doppler radar, Bound.-Lay. Meteorol., 49, 331-337, 1989.

Harris, M., Bryce, D. J., Coffey, A. S., Smith, D. A., Birkemeyer, J., and Knopf, U.: Advance measurement of gusts by laser anemometry, 95, 1637-1647, 2007.

Kindler, D., Oldroyd, A., Macaskill, A., and Finch, D.: An eight month test campaign of the Qinetiq ZephIR system: Preliminary results, Meteorol. Z., 16, 479-489, 2007.

Lee, X.: On micormeteorological observations of surface-air exchange over tall vegetation, Agr. Forest Meteorol., 91, 39-49, 1998.

Lenschow, D. H., Savic-Jovcic, V., and Stevens, B.: Divergence and Vorticity from Aircraft Air Motion Measurements, J. Atmos. Ocean. Tech., 24, 2062-2072, 2007.

Lothon, M., Lenschow, D. H., and Mayor, S. D.: Coherence and scale of vertical velocity in the convective boundary layer from a Doppler lidar, Bound.-Lay. Meteorol., 121, 521-536, 2006.

Mann, J., Cariou, J.-P., Courtney, M. S., Parmentier, R., Mikkelsen, T., Wagner, R., Lindelöw, P., Sjöholm, M., and Enevoldsen, K.: Comparison of 3D turbulence measurements using three staring wind lidars and a sonic anemometer, Meteorol. Z., 18, 135-140, 2009.

Pena, A., Gryning, S., and Hasager, C.: Measurements and modelling of the wind speed profile in the marine atmospheric boundary layers, Bound.-Lay. Meteorol., 129, 479-495, 2008a.

Pena, A., Hasager, C., Gryning, S., Antoniou, I., and Mikkelsen, T.: Offshore wind profiling using light detection and ranging measurements, Wind Energy, 12, 105-124, 2008b.

Raupach, M., Finnigan, J., and Brunet, Y.: Coherent eddies and turbulent in vegetation canopies: the mixing layer analogy, Bound.Lay. Meteorol., 78, 351-382, 1996.

Siegman, A.: Lasers, University Science Books, 1256 pp., 1986.

Sjöholm, M., Mikkelsen, T., Mann, J., Enevoldsen, K., and Courtney, M.: Time series analysis of continuous-wave coherent Doppler Lidar wind measurements, Meteorol. Z, 18, 281-287, 2009.

Smith, D. A., Harris, M., Coffey, A. S., Mikkelsen, T., Jørgensen, H. E., Mann, J., and Danielian, R.: Wind lidar evaluation at the Danish wind test site Høvsøre, Wind Energy, 9, 87-93, 2006.

Sonnenschein, C. M. and Horrigan, F. A.: Signal-to-noise relationships for coaxial systems that heterodyne backscatter from atmosphere, Appl. Optics, 10, 1600, 1971. 